\title{
A lightweight and support-free design method for selective laser melting
}

\author{
Zhonghua Li $^{1,2}$ • David Zhengwen Zhang ${ }^{1,2}$ • Peng Dong ${ }^{2,3}$ • Ibrahim Kucukkoc ${ }^{2,4}$
}

Received: 9 July 2016 / Accepted: 18 September 2016/Published online: 25 October 2016

(C) The Author(s) 2016. This article is published with open access at Springerlink.com

\begin{abstract}
Topology optimisation is an effective approach to design extreme lightweight components. However, most of the resulted optimum lightweight components usually have complex structures which cannot be produced successfully by traditional manufacturing processes. Selective laser melting is one of the additive manufacturing processes. It shows powerful capacity in the manufacturing of metal components with complex structures. Therefore, the combination of topology optimisation and selective laser melting shows a promising prospect for metal components. However, support structures were usually introduced during the selective laser melting manufacturing process, which resulted to some disadvantages, for example, the support structures are generally difficult to remove from the original components because it is difficult to clamping and machining. Therefore, a design method was proposed in this
\end{abstract}

David Zhengwen Zhang

zhangzw@cqu.edu.cn

Zhonghua Li

lizhonghua6868@163.com

Peng Dong

dp830130@163.com

Ibrahim Kucukkoc

I.Kucukkoc@exeter.ac.uk

1 The State Key Laboratory of Mechanical Transmissions, Chongqing University, Chongqing 400044, China

2 College of Engineering, Mathematics and Physical Sciences, University of Exeter, North Park Road, Harrison Building, Exeter EX4 4QF, UK

3 Capital Aerospace Machinery Company, Beijing 100076, China

4 Department of Industrial Engineering, Balikesir University, Cagis Campus, Balikesir, Turkey study, named lightweight and support-free design method, the detailed design process and advantages were presented. In the design process, topology optimisation was applied to realise lightweight design, and support-free design process was developed to meet the support-free requirement. Finally, as a case, a cross-beam component was designed using the proposed method, and the final model was produced successfully using selective laser melting process. The case study result verified that the proposed design method is effective to design lightweight and support-free industrial metal components for SLM process.

Keywords Selective laser melting · Topology optimisation • Lightweight $\cdot$ Support-free

\section{Introduction}

Topology optimisation (TO) is a mathematical method that distributes materials optimally within a given design domain, based on specific loads and boundary conditions. It is an effective approach to reducing weight for component design. However, most of the optimum lightweight components from TO have complex structures, which usually cannot be manufactured using traditional manufacturing processes. Sometimes, it is infeasible to manufacture complex structures because of the need for tool access space in machining or removing of the dies in casting [5]. Selective laser melting (SLM) is an additive manufacturing (AM) technology, which can manufacture metal components directly from three dimensional (3D) models. In SLM processes, components are manufactured selectively by fusing and consolidating metal powder layer-by-layer via a computer-controlled scanning laser beam. Therefore, SLM shows a high potential for manufacturing metal components with complex structures which cannot be manufactured by traditional processing technologies [4]. 
Because of the complexity and intricacy of the solutions obtained, TO was often constrained to research and theoretical studies. However, SLM as an additive manufacturing process, fills the gap between TO and metal application. Therefore, the combination of TO and SLM shows a promising prospect for the optimum lightweight metal components. Many researches have been carried out to study the combination of TO with AM technologies. For example, Brackett et al. [5] summarised the main challenges and opportunities of TO for AM. Brackett et al. [5] emphasised that the applicability of TO for AM will develop and increase rapidly. Chahine et al. [7] designed porous structures using TO and manufactured them using Electron Beam Melting (EBM). Aremu et al. [2] investigated the effects of starting design, finite element mesh and parametric values on optimum structure achieved by Solid Isotropic Material with Penalisation (SIMP) and bi-directional evolutionary structural optimisation (BESO) algorithms, since they have been widely implemented to achieve practical designs. Xiao et al. [20] explored the maximum stiffness of microstructure with the constraint of volume fraction by TO method and produced metallic biomaterial scaffolds by SLM technology. Zegard and Paulino [21] explored the applicability of two TO methods, i.e. the ground structure method and the density-based method, for the optimal design structures produced using FDM and SLS technologies. Aremu et al. [3] studied the effect of TO design parameters on the performance of an additively manufactured part. The EADS (European Aeronautic Defence and Space Company) Innovation Works and EOS (a German SLM systems and service supplier) cooperated in the manufacture of Airbus A320 and A380 brackets with optimised topology. The results showed an obvious reduction in weight and raw material consumption $[12,18,22]$. However, the majority of the studies on TO and SLM focused on micro-lattice structured parts. Only few designs focused on the macrostructure parts. One great obstacle is that the support structures are generally imported in the SLM process as there are some technical constraints and design rules for SLM, such as the minimum feature size, the manufacturing inclination angle and the allowable overhang distance $[8,10]$. Some studies have been conducted to investigate design constraints. Thomas [17] developed a set of design rules to allow for predictable and reliable results when manufacturing parts using SLM process. Kranz et al. [9] investigated the influence of part position and orientation on the dimension accuracy and surface quality, thin walls, bars and bore holes with varying diameters were built in different orientations to determine the process limit. Wang et al. [19] studied the design rules, including the critical inclined angle, thin walls and cylinders, in order to fabricate the porous structure precisely based on SLM. Adam and Zimmer [1] developed design rules within the project "direct manufacturing design rules" for three additive manufacturing processes, namely SLM, SLS and FDM.

Support structures are required in several AM processes (such as SLM and EBM) to sustain overhanging parts, in particular for the production of metal components [16]. For
SLM process, support structures are designed to be withstand the thermal stress at locations where it could cause damage on the part. The main shortcomings resulted by the support structures can be summarised as follows:

- The support structures are difficult to remove from the original part. This is due to the final optimum lightweight model resulted from TO usually have a complex structure, which is not suitable for clamping and machining.

- The total manufacturing time increases because (i) a preprocessing time is required to generate support structures, (ii) a SLM processing time is required to manufacture support structures and (iii) a post-processing time is required to remove the support structures.

- The total cost increases as the support structures need extra material and SLM processing time, in addition to the preprocess and post-process.

Since the abovementioned reasons, the design of support-free components for SLM technology will be a hotspot in near future. So far, extremely little attention was paid to this topic. Leary et al. [10] presented a strategy for minimising the support material use by comparing the feasible limits of FDM manufacture to the build angles that exist within a proposed geometry. Leary et al. [11] proposed a method which modifies the theoretically optimal topology as required to enable support-free AM. A case was analysed using fused deposition modelling (FDM) process. Calignano [6] investigated the manufacturability of overhanging structures using optimised support parts and performed an experimental study to identify the optimal support-free overhanging structures using Taguchi $L_{36}$ design. Seabra [15] optimised an aircraft bracket and manufactured it by means of SLM using the TO method. The optimised component showed considerable weight reduction with an increase of the safety factor. However, as shown in Fig. 1, the final structure was not support free which needed post-processing to remove the support structures.

To the best of authors' knowledge, no research was conducted on the simultaneous lightweight and support-free

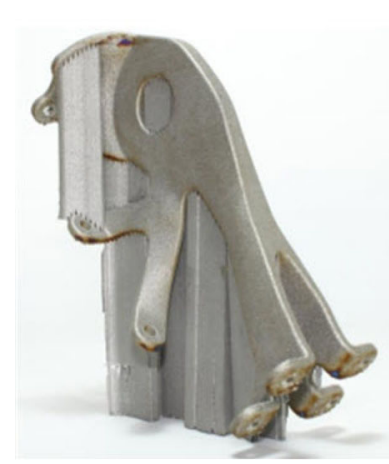

(a)

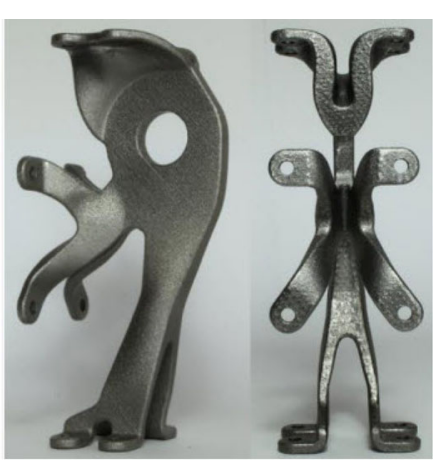

(b)
Fig. 1 Optimised aircraft bracket a with support structures, b removal of the support structures [15] 
design of industrial metal components for the SLM process. This study proposes a simultaneous lightweight and supportfree (L\&S) design method for SLM and presents its design details for the first time in the literature. As a real-world application, a cross-beam structure component is designed using the proposed method and manufactured using the SLM technology. The case study verifies the effectiveness of the proposed L\&S design method for SLM process. Thus, this study shows how metal components can be produced using SLM consuming less material and requiring no post-processing operations.

The remainder of this paper is organised as follows. Section 2 describes the L\&S design method proposed. Section 3 presents a case study on designing an L\&S crossbeam structure using the proposed method, and manufacturing it using the SLM technology. A discussion is also presented in the same section followed by the conclusions and future research directions provided in Section 4.

\section{L\&S design method}

A L\&S design method is proposed in this study in order to design lightweight industrial metal components, which can be produced directly by SLM process without any support structures. In the design process, TO technology is introduced to realise the goal of lightweight and support-free design process was developed in meeting the requirement of support-free.

The $L \& S$ design method is an optimisation process to find optimal lightweight structures that fulfil support-free expectation of SLM technology. The flow chart of the L\&S design method is shown in Fig. 2. The following sub-sections provide the detailed design steps.

\subsection{Analysis of the original part}

The original part is analysed to determine whether the original model needs to be optimised to realise lightweight. It is obvious that every component has its own design constraints, such as the maximum strain, stress, mass and displacement, which can be expressed mathematically as in Equation (1).

$T_{i, j, k, \ldots}(x)<C_{i, j, k, \ldots}(x)$

where $x$ is the design variable and $i, j, k, \ldots$ are different constraint types. The terms $T_{i, j, k}, \ldots(x)$ and $C_{i, j, k}, \ldots(x)$ denote the result functions and the constraint functions, respectively, which may be constant or variable. The possible conditions are as follows:

- $T(x)$ is much smaller than $C(x)$ : The original model needs to be optimised.

- $T(x)$ is equal or close to $C(x)$ : The original model does not need to be optimised.
- $\quad T(x)$ is much larger than $C(x)$ : The original model is an unqualified design which does not need to be optimised.

The finite element method (FEM), also referred to as finite element analysis, is employed to analyse the original model. FEM is an effective computational tool for performing engineering analysis. In practice, FEM usually consists of three principal steps:

(a) Pre-processing: The original models are built and meshed, elements are connected by "nodes". Prescribed constraints and load are applied on the nodes.

(b) Analysis: The dataset prepared by a pre-processor is used as an input to the finite element code itself, which constructs and solves a system of linear or nonlinear algebraic equations. For example, the basic finite element equation to be solved for a structure which experiencing static loads can be expressed as:

$K u=P$

where, $K$ is the stiffness matrix of the structure. The terms $u$ and $P$ denote the displacement vector and external applied loads at certain nodes, respectively.

(c) Post-processing: The results are analysed and expressed in a desired style.

\subsection{Lightweight design}

TO method is employed in this step to optimise the original model to realise lightweight design. TO is a mathematical approach that optimises material layout within a given design space, for a given set of loads and boundary conditions such that the resulting layout meets a prescribed set of performance targets. The characteristic material is distributed in the design space during the TO procedure which results in the best objective function value while preserving all constraints in the problem definition. Using TO, engineers can find the best concept design that meets the design requirements.

Several methods have been proposed for implementing TO in determining material layout on a given design space. Some of those popular ones are SIMP, homogenisation, BESO, level set method, ant colony optimisation algorithm and genetic algorithms [2]. The SIMP approach is selected in the L\&S method due to its advantages over other methods. Firstly, it has been studied extensively and can be applied to complicated problems. Secondly, SIMP requires less computational effort as it has only one design parameter for each element.

Based on the SIMP method, the structure lightweight optimisation problem can be expressed as in Equation 3.

$\operatorname{Min} V(X)=V\left(x_{1}, x_{2}, \ldots, x_{n}\right)$ 
Fig. 2 Flow chart of L\&S design method

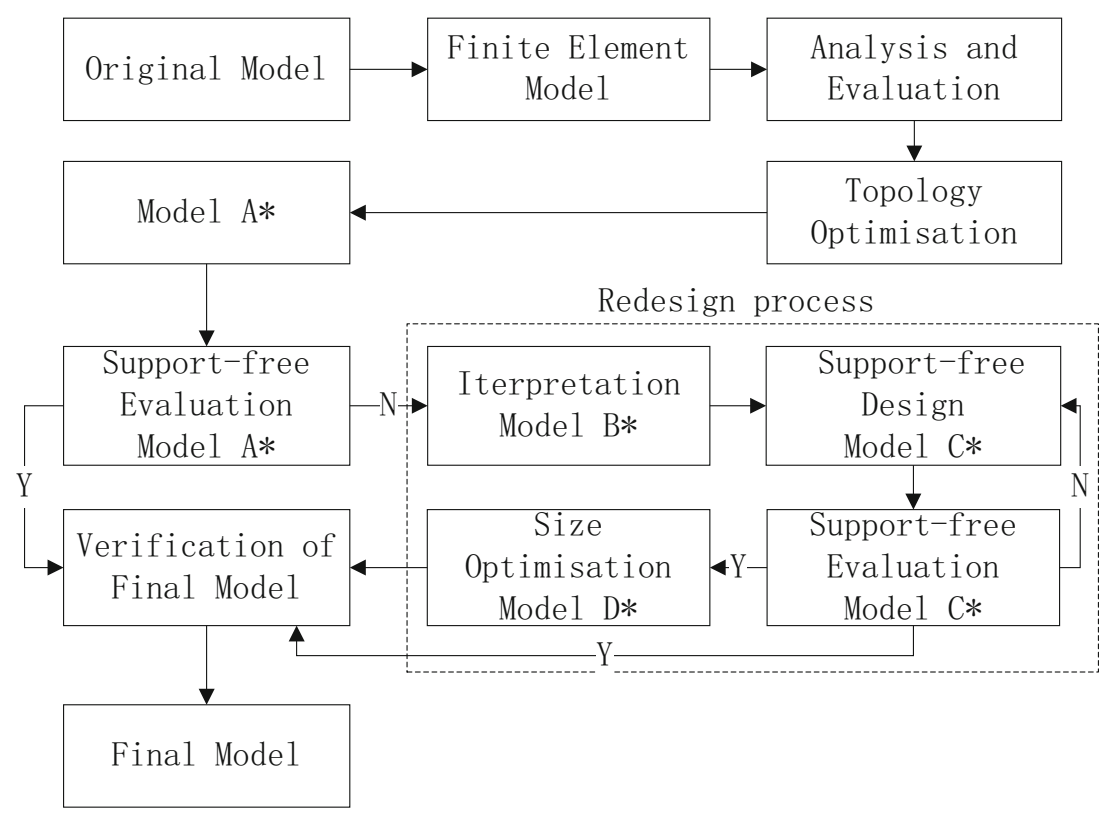

Subject to:

$g(X)<0$

$x_{i}^{L} \leq x_{i} \leq x_{i}^{U} \quad i=1, \ldots, n$

where, $V(X)$ is the objective volume function, $g(X)$ is the constraint function, $x$ is the design variable which represent element densities in TO. $x_{i}$ denotes the $i$-th density design variable where there are a total of $n$ design variables, $L$ and $U$ denote the lower bounds and upper bounds of $x_{i}$, respectively.

The TO model with design varieties, responses, constraints and objective is generated and executed based on the FEM model given in Section 2.1. The optimal design model obtained eventually is named model $\mathrm{A}^{*}$.

\subsection{Support-free evaluation of model $A^{*}$}

The build orientation is influenced by function requirement and assembly relations. Different build orientation will result in different support structure volume, manufacturing time and cost. Therefore, the build orientation should be identified before support-free evaluation of model $A^{*}$. In general, the build orientation can be identified directly by engineer's experience for the part with simple structures. For the parts with complex structures, the optimal orientation should be identified by an optimization algorithm. Please refer to a relevant study performed by Strano et al. [16].

Model $\mathrm{A}^{*}$ is evaluated to determine whether it can be manufactured directly without any support structure using SLM process. Evaluation process is based on the SLM manufacturing constraints, such as the inclination angle, minimum circle diameter and maximum overhang distance, which may vary depending on the machine type and process parameters. For example, let $\alpha_{r}$ and $\alpha_{c}$ be the actual and the constraint inclination angles of model $\mathrm{A}^{*}$, respectively. If $\alpha_{r} \geq \alpha_{c}$, no support structure is required; otherwise, support structures need to be built.

If model $\mathrm{A}^{*}$ satisfies all of the following main SLM manufacturing constraints, it is considered as the final supportfree model, which has the optimum lightweight. Otherwise, it needs to be designed to satisfy following conditions:

$\alpha_{r} \geq \alpha_{c}$

$D_{c l} \leq D_{r} \leq D_{c u}$

$O_{r} \leq O_{c}$

$T_{r} \geq T_{c}$

where, $D_{c l}, D_{r}$ and $D_{c u}$ are the lower bound, actual and upper bound values for the diameter of the support-free circle, respectively. $O_{r}$ and $O_{c}$ are the actual and constraint values of the overhanging distance, respectively. $T_{r}$ and $T_{c}$ denote the actual and constraint values of the thickness of shell or beam, respectively.

If model $\mathrm{A}^{*}$ has a simple structure, it can be evaluated directly by the designer according to the SLM manufacturing constraints. If it has a very complex structure, a commercial software can be employed for the evaluation process, such as Magics 13.0 [14]. The models obtained from the TO usually have irregular narrow surfaces, which cannot be identified using a software. Therefore, the evaluation process usually requires experienced designers.

\subsection{Interpretation of model $A^{*}$}

Usually, model $\mathrm{A}^{*}$ resulted from the TO cannot be edited using a modelling software. Therefore, a 3D solid model, 
named model $\mathrm{B}^{*}$, is built according to the outlines of model A*.

The interpretation process contains two main steps: (i) Measuring of model $\mathrm{A}^{*}$ and (ii) modelling of model $\mathrm{B}^{*}$. Due to the following main reasons, model $\mathrm{B}^{*}$ cannot be exactly the same as model $\mathrm{A}^{*}$. Firstly, the model obtained from TO (model $\mathrm{A}^{*}$ ) usually has many irregular surfaces, which cannot be measured exactly. Secondly, small and narrow surfaces do not need to be built because this will need large amount of modelling time in this step and support-free design time in the following step. Therefore, model $\mathrm{B}^{*}$ must have a slight increase in size and volume in order to confirm the stiffness of the part.

\subsection{Support-free design of model B*}

In this step, some material is added on model B* according to the SLM manufacturing constraints given in Section 2.3 to realise support-free requirement. The support-free model resulted from this process is named model $\mathrm{C}^{*}$.

\subsubsection{Support-free design process}

As shown in Fig. 3b, the actual inclination angle $\alpha_{r}$ is much less than the allowable angle $\alpha_{c}$. If it is manufactured directly without any further design, support structures are required for the SLM manufacturing process in the area enclosed by the blue line (see Fig. 3a). However, the design can be converted into a support-free design by adding as little material as possible in the blue line enclosed area according to the SLM design constraints. The detailed design process is as follows. Firstly, the area enclosed by the blue line is minimised according to the minimum inclination angle $\alpha_{c}$, given in Fig. $3 \mathrm{~b}$. Afterwards, the enclosed area is designed using $\alpha_{c}$ and minimum thickness of shell or beam (represented by $T_{c}$ ). As in
Fig. $3 \mathrm{c}$, the red lines are built in sequence with the same inclination angle of $\alpha_{c}$. These lines represent the beam with the minimum thickness of $T_{c}$, which may vary at different angles and heights. The design area is reduced to the yellow areas and beams, which cannot be changed later. The yellow areas are usually narrow and it is less effective to design these areas in this step. Therefore, the yellow areas are designed using circles and ellipses, which are tangent to the lines (see Fig. 3d). As discussed in Section 2.3, support-free circle should conform to the constraint of $D_{c l} \leq D_{r} \leq D_{c u}$. A support-free ellipse is developed based on the support-free circle, as will be explained in Section 2.5.2.

In a narrow space, the allowable maximum support-free circle and support-free ellipse are certain. The one which has the larger area is selected so that more material can be saved.

\subsubsection{Support-free design of ellipse}

Support-free ellipses are developed on the basis of the support-free circles. The equation of an ellipse whose major and minor axis coincide with the Cartesian axis can be expressed as follows.

Major axis coincides with $\mathrm{x}$ axis:

$\frac{x^{2}}{a^{2}}+\frac{y^{2}}{b^{2}}=1$

Major axis coincides with y axis:

$\frac{y^{2}}{a^{2}}+\frac{x^{2}}{b^{2}}=1$

where, $a$ is the distance of semi-major axis and $b$ is the distance of semi-minor axis.
Fig. 3 Support-free design process

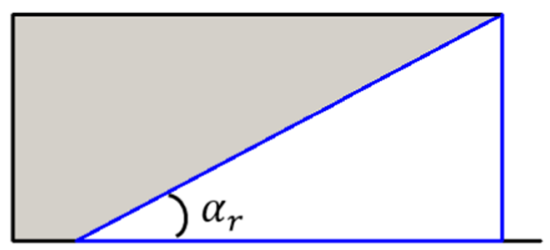

(a)

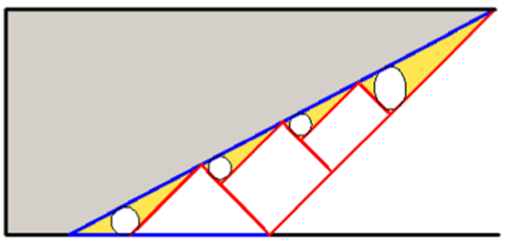

(d)

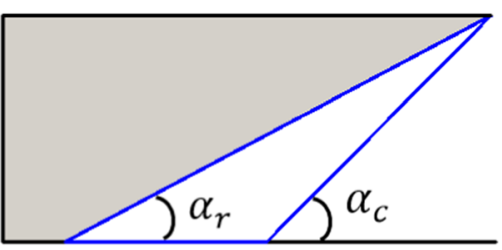

(b)
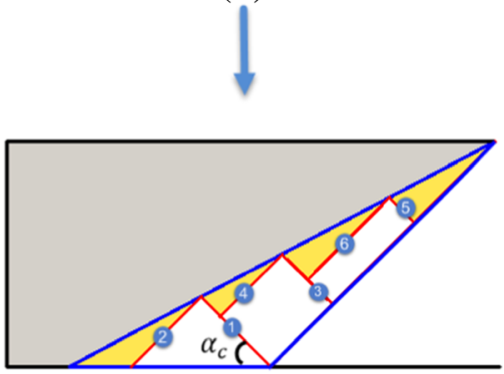

(c) 
The equation of a circle whose axis coincide with the Cartesian axis can be expressed as:

$x^{2}+y^{2}=R^{2}, \quad R=\frac{1}{2} D$

where, $R$ and $D$ are the radial and diameter of the circle, respectively.

The support-free ellipses whose major axis coincides with $\mathrm{x}$ axis cannot be derived from the support-free circles. This is because there is no definite relationship between $\Phi$ (the angle of tangent line with level for ellipse) and $\Psi$ (the angle of tangent line with level for support-free circle). Therefore, the support-free ellipses whose major axis coincides with $\mathrm{x}$ axis should be obtained from the SLM experiments. In this study, only the ellipse whose major axis coincides with y axis is developed.

As shown in Fig. 4, the circles $D_{c l}$ and $D_{c u}$ are the lower and upper bound diameters of support-free circles $O 1$ and $O 2$, respectively. For the ellipse whose major axis coincides with y axis, the support-free ellipse should satisfy any one of the following constraints:

(a) $\frac{1}{2} D_{c l} \leq b \leq a \leq \frac{1}{2} D_{c u}$ as in Fig. $5 \mathrm{a}$

(b) $\frac{1}{2} D_{c l} \leq b \leq \frac{1}{2} D_{c u} \leq a$, as in Fig. 5b

(c) when a $>b>\frac{1}{2} D_{c u}$, points $P$ and $Q$ are below points $M$ and $N$, as in Fig. $5 \mathrm{c}$. Move the circle $O 2$ up to realise points $H$ and $H^{\prime}$ coincide, where points $P$ and $Q$ are the intersection points of ellipse $O$ and circle $O 2 . L$ is the tangent line of circle $O 2$, the angle of $L$ with level line is the constraint angle $\alpha_{c}$, where $M$ and $N$ are the tangent points.

\subsection{Size optimisation of model $C^{*}$}

It is obvious that model $\mathrm{C}^{*}$ is heavier than $\mathrm{A}^{*}$ and $\mathrm{B} *$, because additional material was added in the interpretation and support-free design steps. Size optimisation defines ideal

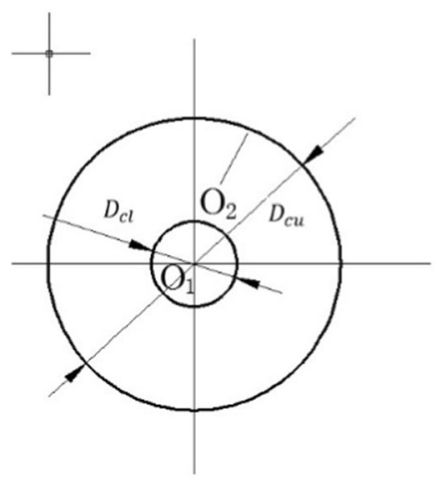

(a)

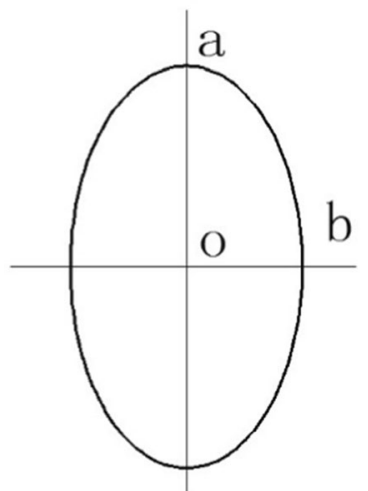

(b)

Fig. 4 Constraints for support-free ellipse

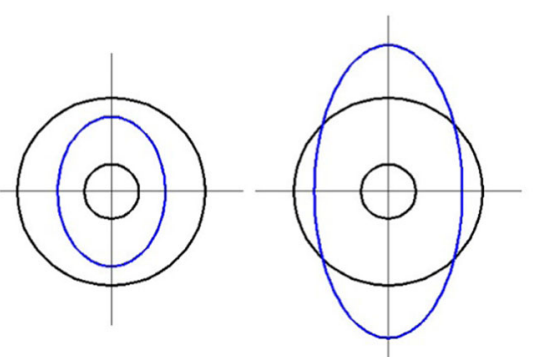

(a)

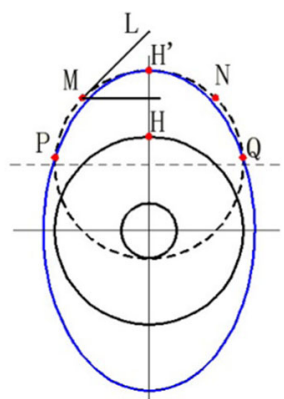

(c)
Fig. 5 Support-free ellipse in different conditions

component parameters, such as cross-section dimensions and thicknesses. If model $C^{*}$ conforms to size optimisation rules, it is further optimised using size optimisation technique and the final model (called model $\mathrm{D}^{*}$ ) is eventually obtained. Otherwise, model $\mathrm{C}^{*}$ is considered as the final model. As the size optimisation technique is a widely used well-known optimisation method, the detailed optimisation process will not be repeated here.

\subsection{Verification of the final model}

FEM is used in this step to make sure that the final model satisfies the design objectives and constraints. Afterwards, the final model is manufactured using SLM to verify that it can be produced successfully without any support structures.

\section{Case study}

The aims of this section are to design an L\&S cross-beam structure using the proposed method and to manufacture it using SLM technology.

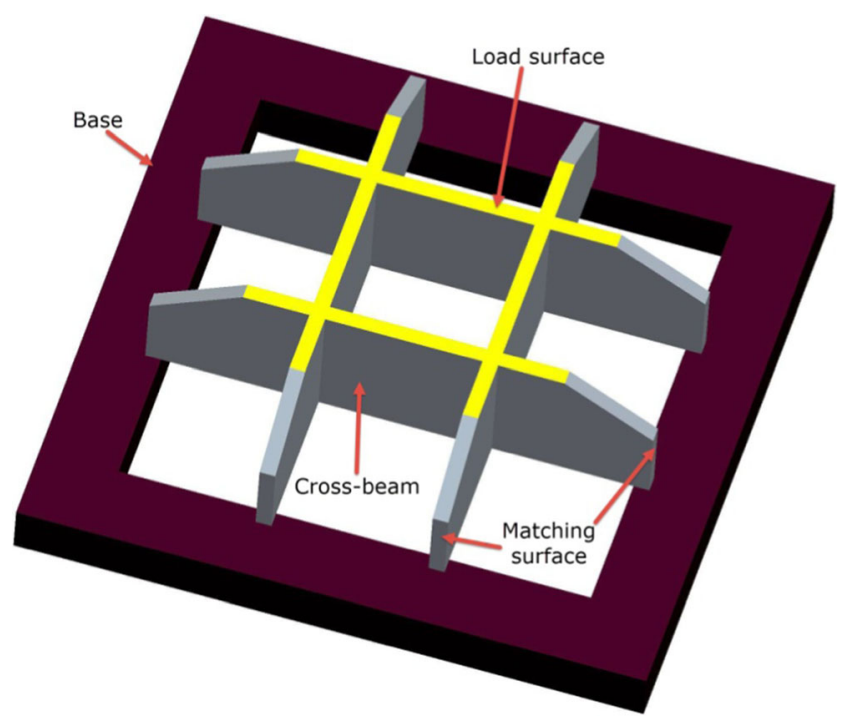

Fig. 6 Original cross-beam structure 


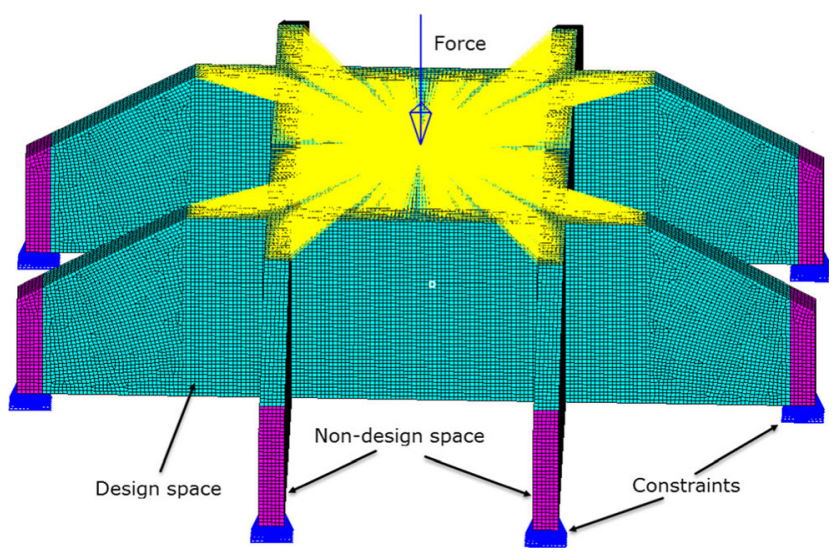

Fig. 7 Load and constraint conditions of cross-beam structure

The cross-beam structure is made of steel and fixed to the base structure by welding, as shown in Fig. 6 . The original component is assembled using riveting and thread-connecting processes from eight parts, which were produced by traditional processing technologies. Therefore, the structure of the original component is largely defined by the traditional processing technologies. Its design is simple and imaginable for designers. Although it can be manufactured directly without any support structures using SLM process, it is obvious that the cross-beam structure does not have an ideal lightweight structure.

\subsection{Analysis of original part}

The original part was analysed through FEM using commercial software, Altair HyperWorks 13.0. The original crossbeam component is a support bracket which has a vertical load on the top surface, as shown in Fig. 7. The solid model is meshed with a hexahedral element and divided into two spaces: design space and non-design space. The eight square red pillars show the non-design space, which will be fixed to the base by welding and matched with other parts. The blue part is the design space, which will be optimised and designed to have a lightweight and support-free structure. The constraints were applied at the bottom surface of the eight nondesign spaces, so that each node was constrained fully. RBE 3 element was put on the top surface of the component in order to distribute the force on all nodes uniformly and the load was applied on the RBE 3 element. The material properties of steel, including elastic modulus, Poisson's ratio and density were used for both design and non-design spaces, as shown in

Table 1 Material properties of steel

\begin{tabular}{lc}
\hline Properties & Parameter \\
\hline Elastic modulus (E) & $2.1 \mathrm{E} 5(\mathrm{MPa})$ \\
Poisson's ratio (NU) & 0.3 \\
Density (RHO) & $8.0 \mathrm{E}-9\left(\mathrm{t} / \mathrm{mm}^{3}\right)$
\end{tabular}

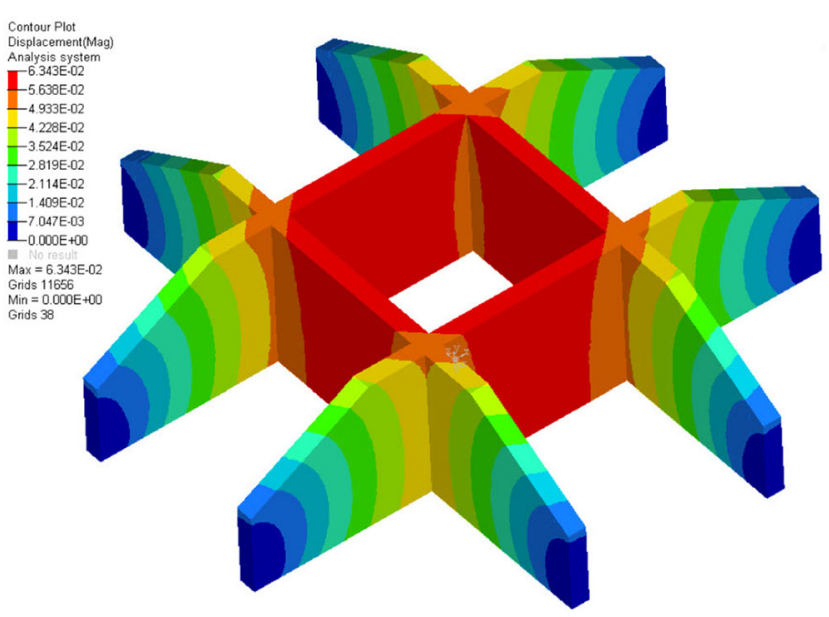

Fig. 8 FEM analysis of original component

Table 1. A maximum displacement of $0.2 \mathrm{~mm}$ was allowed in the final structure, the thickness of the beam can not be changed.

When FEM was conducted, the maximum displacement was observed as $0.063 \mathrm{~mm}$ (Fig. 8), which is much less than the allowable displacement of $0.2 \mathrm{~mm}$. This indicates that there is a significant amount of inefficient material in the original structure.

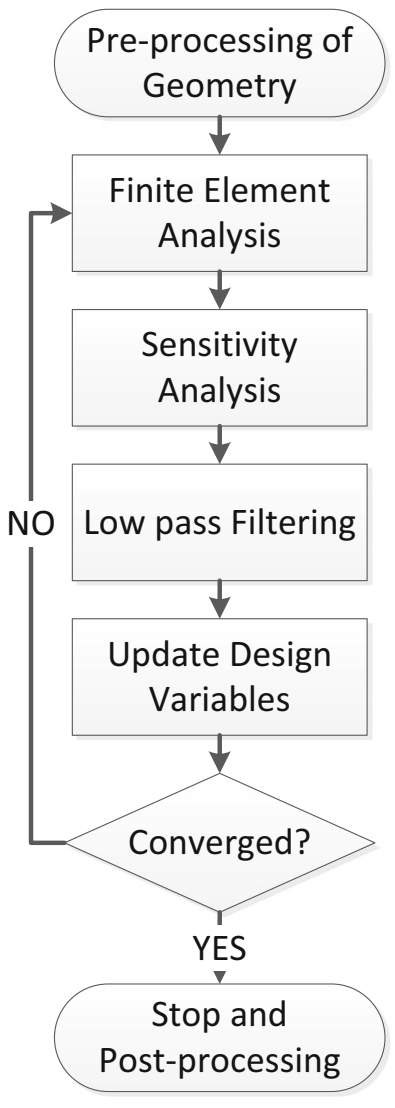

Fig. 9 Flow chart of TO process 
Table 2 Displacements at different threshold values

\begin{tabular}{llllllll}
\hline Threshold value & 0.10 & 0.20 & 0.30 & 0.40 & 0.31 & 0.32 & 0.33 \\
\hline Displacement & 0.135 & 0.157 & 0.186 & 0.238 & 0.191 & 0.197 & 0.204
\end{tabular}

\subsection{Lightweight design}

The TO parameters were set up (including design variables, responses, constraints and objectives) based on the FEM, and the SIMP-based TO was performed. A feasible design was obtained from the TO, of which the flow chart is presented in Fig. 9.

Various models with different threshold values were analysed to determine the minimum part volume under the allowable displacement constraint. The threshold value is a constant between 0 and 1 . The smaller the threshold value, the larger the final extracted volume. In the contrary, the smaller the final volume, which companied with a lower stiffness and strength. Therefore, the selection of threshold value is crucial for the final design. The selection procedure of threshold value is as follows. Starting from 0.1, the threshold value is increased by 0.1 step-wise and different models were executed through FEM. When the threshold value was increased to 0.4 , the maximum displacement of $0.218 \mathrm{~mm}$ was generated, which is over the allowable displacement of $0.2 \mathrm{~mm}$. Therefore, the reasonable threshold value is between 0.3 and 0.4 . This time, the threshold value is increased by 0.01 starting from 0.31 and FEM was conducted accordingly until the maximum displacement of $0.204 \mathrm{~mm}$ was obtained at the threshold value of 0.33 . So it was concluded that the reasonable threshold value was 0.32 . The threshold values observed during this process are presented in Table 2. Model $\mathrm{A}^{*}$, with 0.32 density, was exported in STL format as in Fig. 10 .

\subsection{Support-free evaluation of model A*}

The build orientation was identified as in Fig. 11 because the top surface of the part is the loading surface which to be

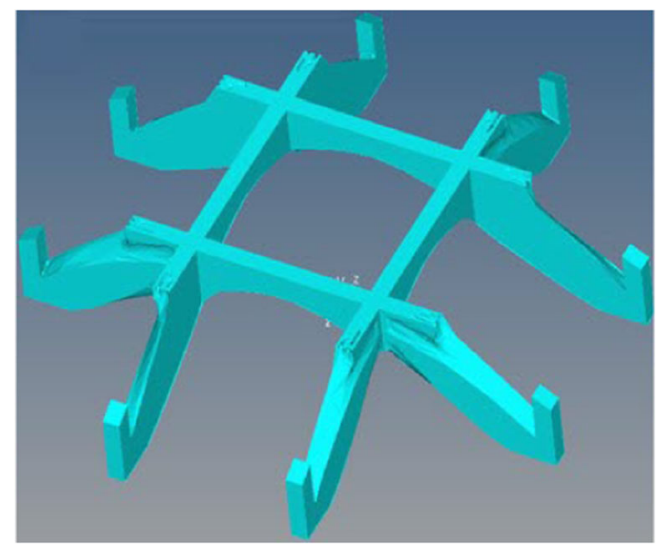

Fig. 10 Model A* in STL format at 0.32 density

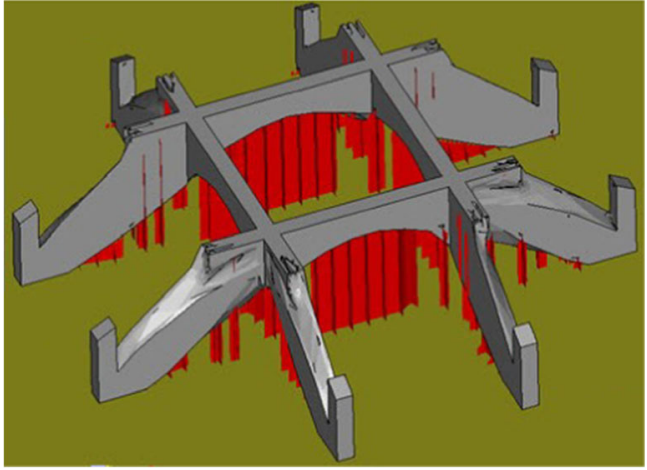

Fig. 11 Model A* with support structures

assembled with other components. If it was built upside down, the assembly process could be disturbed because additional structure was designed on the top surface.

Support-free evaluation was performed using the SLM manufacturing constraints listed in Section 2.3. As model $A^{*}$ is very complex, the commercial software, Magics 13.0, was employed in this step, which can be used in all kinds of additive manufacturing processes to build support structures and calculate the volume for the part. As shown in Fig. 11, model A* was generated with support structures. The red space corresponds to support structures which are required if the cross-beam part is manufactured directly using model A*. Therefore, according to the L\&S method, model A* needs to be redesigned to be support-free.

\subsection{Interpretation of model A*}

In this step, model $\mathrm{A}^{*}$ was interpreted into a $3 \mathrm{D}$ solid model (model $\mathrm{B}^{*}$ ) using a modelling software, Pro/Engineer 4.0. In order to confirm the stiffness of the part, the interpreted model usually has a slight increase in size and volume [13]. As shown in Fig. 12, model $\mathrm{B}^{*}$ was built as the measurements gathered from model $\mathrm{A}^{*}$. The final model $\mathrm{B}^{*}$ is shown in Fig. 13a. The support structures of model $\mathrm{B}^{*}$ were generated by Magics 13.0, as in Fig. 13b, which is similar to the support structures of model $\mathrm{A}^{*}$. The volumes of model $\mathrm{A}^{*}$ and model B* were measured $95,682.425 \mathrm{~mm}^{3}$ and $110,114.4 \mathrm{~mm}^{3}$, respectively, which indicate that the interpretation design was reasonable.

\subsection{Support-free design of model B*}

The objective of this step was to design model $\mathrm{B}^{*}$ using the method developed in Section 2.5. As shown in Fig. 14a, the minimum inclination angle of $\alpha_{c}$ and the minimum thickness of $T_{c}$ were used to define the overall material distribution in the blue line enclosed area. The support-free ellipses were designed in the narrow space according to the design rules developed in Section 2.5.2. The model obtained in this step (model $\mathrm{C}^{*}$ ) is shown in Fig. 14b. Fig. 14c shows model $\mathrm{C}^{*}$ 
Fig. 12 Interpretation of model $\mathrm{A}^{*}$

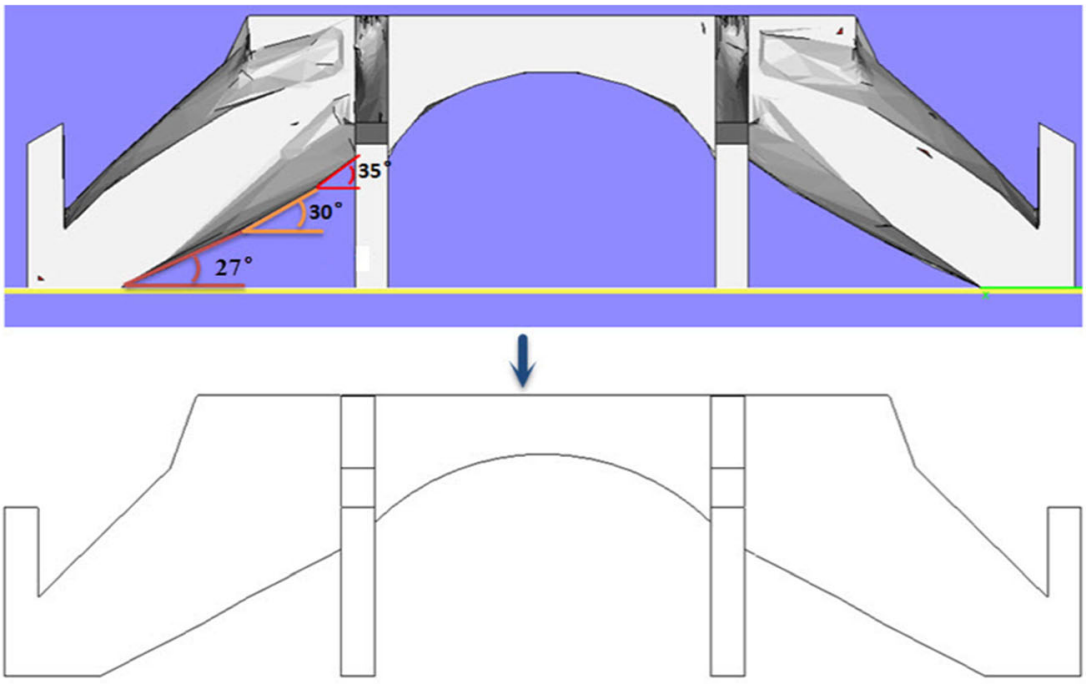

with support structures. It is obvious that support structures are not required, which indicate that the developed design method for support-free was effective. As model $C^{*}$ violates its size constraints, it was considered as the final model.

\subsection{Verification and manufacturing of final model}

In this step, FEM was conducted in order to verify that model $\mathrm{C}^{*}$ meets the design constraints. Model $\mathrm{C}^{*}$ was meshed using tetrahedral element as the final part was very complicated. The FEM process was conducted and the results are shown in Fig. 15a. The maximum displacement does not go beyond the constraints of $0.2 \mathrm{~mm}$, which indicates that the proposed L\&S design method is effective. As shown in Fig. 15b, the final model was produced successfully without any support structures using SLM technology.

\subsection{Results and discussion}

As can be seen from Fig. 15a, only $0.103 \mathrm{~mm}$ of maximum displacement was found, which still greatly lower than the allowable displacement of $0.2 \mathrm{~mm}$. This is due to the large increase of the final model volume compared with the initial

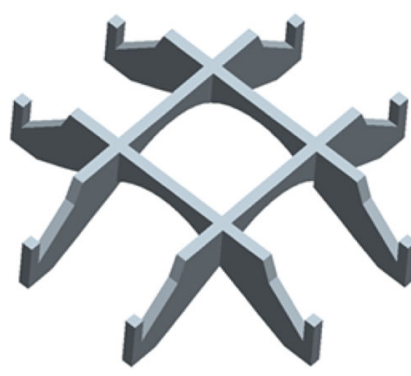

(a)

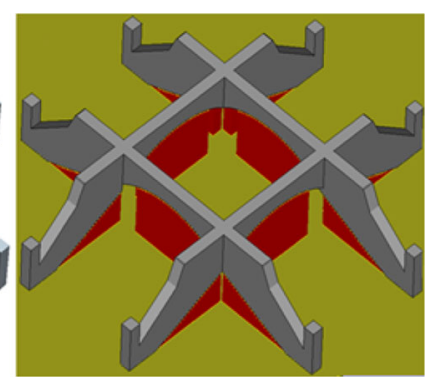

(b)
Fig. 13 a Model B* and $\mathbf{b}$ its support structures optimisation result. There are two main steps resulted in the large volume increase: interpretation step and support-free design step. Table 3 shows the volumes at different design stages. It is obvious that $\mathrm{TO}$ is an effective way to remove material from the original part, $52.2 \%$ of the volume of the original part was removed. The volume of model $\mathrm{B}^{*}$ increased slightly (about $7.2 \%$ ) compared with model $\mathrm{A}^{*}$ in the interpretation process in order to confirm the stiffness of the part. The volume of model $\mathrm{C}^{*}$ increased dramatically owing to material being added in order to realise a support-free design. A $13.6 \%$ volume increase was observed compared with model $\mathrm{B}^{*}$, which indicates that support-free design process is critical for the lightweight design of the part.

\section{Conclusions and future work}

A new lightweight and support-free design method for selective laser melting was proposed, named L\&S design method. The detailed design process which includes two main steps was presented. Initially, TO was employed to optimise the parts to be lightweight. Then, support-free design process was developed to enable producing parts without any support structures. The SLM manufacturing constraints were introduced, and support-free ellipse was developed building work over the support-free circle. The proposed L\&S design method enables the SLM process to present its high potential capacity in the manufacturing of lightweight and complex structures. At the same time, additional advantages can be drawn as follows: the parts designed by L\&S method do not need post processing to remove support structures; the total cost and total manufacturing time (including pre-processing time, manufacturing time and post-processing time) have been minimised.

As a case study, a cross-beam component was designed using the L\&S design method. The model that was generated finally 
Fig. 14 a Support-free design of model B*, b model $\mathrm{C}^{*}$ and $\mathbf{c}$ support stage of model $\mathrm{C}^{*}$

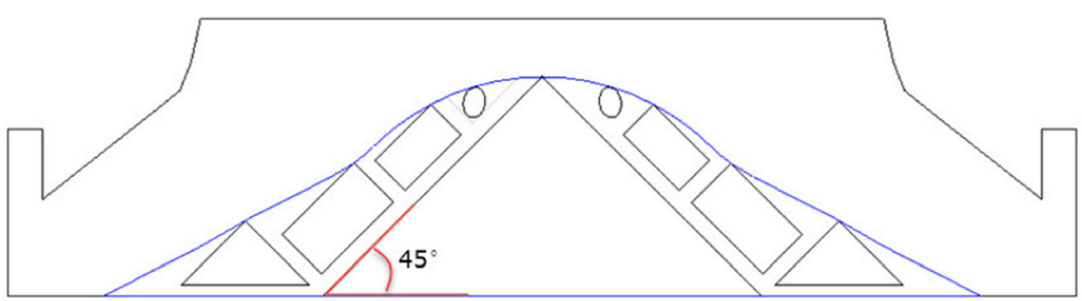

(a)

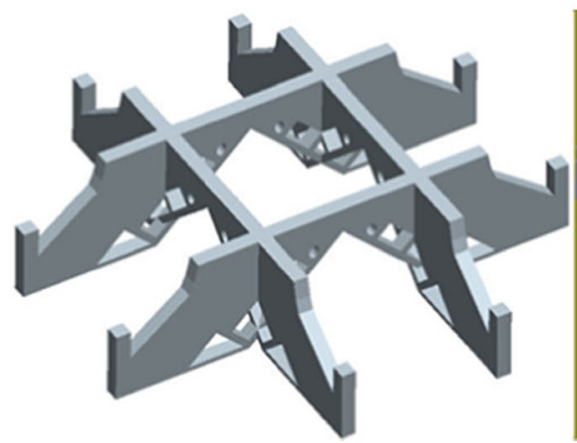

(b)

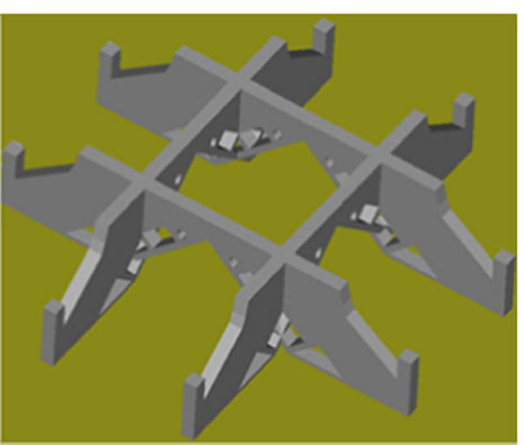

(c)

Fig. 15 a FEM of final model, b physical model

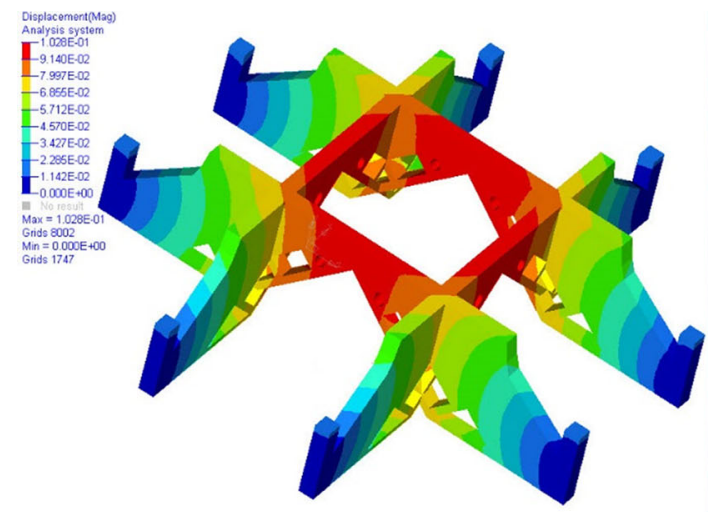

(a)

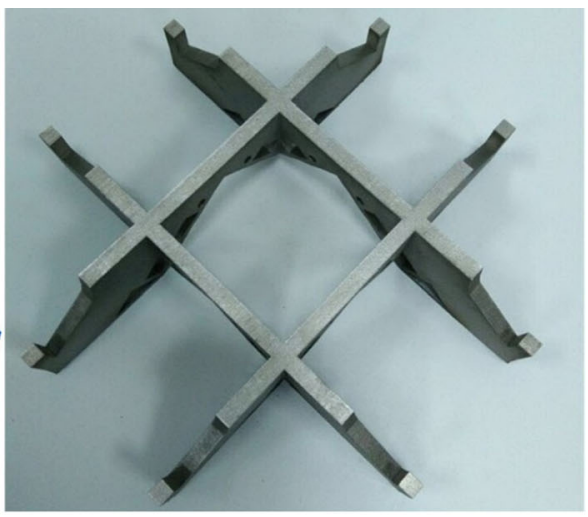

(b) has a $31.4 \%$ volume reduction compared with the original part. To show the effectivity of the L\&S design method, the final model was manufactured directly using SLM technology without any support structures. In terms of the potential applications of the study, the proposed L\&S design method can be used in industry for manufacturing L\&S parts by SLM technology, especially in aerospace field which has a high demand for lightweight components. Also, the support-free design method developed in this study is suitable for the two dimensional design. One limitation of the study is that, a $20.8 \%$ volume increase has occurred in the redesign process, of which $13.6 \%$ volume increase belongs to the support-free design process. Therefore, more efforts should be devoted in the redesign process (and also the interpretation and support-free design steps) to keep the increase in volume at a minimum.
Table 3 Evaluations at different design stages

\begin{tabular}{clcll}
\hline Models & Volume $\left(\mathrm{mm}^{3}\right)$ & Volume Reduction $(\%)$ & Volume of support structures & Post processing \\
\hline Original & $200,313.4$ & N/A & 0 & No \\
Model A* & $95,682.4$ & 52.2 & - & Yes \\
Model B* & $110,114.4$ & 45.0 & $42,346.1$ & Yes \\
Model C* & $137,491.4$ & 31.4 & 0 & No \\
\hline
\end{tabular}


Acknowledgments This research was supported by the National High Technology Research and Development Program of China (863 Program: 2015AA042501). All authors thank to the valuable comments and suggestions of the editors of The International Journal of Advanced Manufacturing Technology and the anonymous referees which helped improve the presentation and quality of the manuscript.

Open Access This article is distributed under the terms of the Creative Commons Attribution 4.0 International License (http:// creativecommons.org/licenses/by/4.0/), which permits unrestricted use, distribution, and reproduction in any medium, provided you give appropriate credit to the original author(s) and the source, provide a link to the Creative Commons license, and indicate if changes were made.

\section{References}

1. Adam GAO, Zimmer D (2014) Design for additive manufacturing-element transitions and aggregated structures. CIRP J Manuf Sci Technol 7(1):20-28. doi:10.1016/j.cirpj.2013.10.001

2. Aremu A, Ashcroft I, Hague R, Wildman R, Tuck C (2010) Suitability of SIMP and BESO topology optimization algorithms for additive manufacture. Paper presented at the 21st Annual International Solid Freeform Fabrication Symposium (SFF)-An Additive Manufacturing Conference

3. Aremu A, Ashcroft I, Wildman R, Hague R, Tuck C, Brackett D (2013) The effects of bidirectional evolutionary structural optimization parameters on an industrial designed component for additive manufacture. Proc Inst Mech Eng B J Eng Manuf 227(6):794-807. doi:10.1177/0954405412463857

4. Bhavar V, Kattire P, Patil V, Khot S, Gujar K, Singh R (2014) A review on powder bed fusion technology of metal additive manufacturing. Paper presented at the 4th International Conference and Exhibition on Additive Manufacturing Technologies-AM-2014, September 1-2, Bangalore, India

5. Brackett D, Ashcroft I, Hague R (2011) Topology optimization for additive manufacturing. Paper presented at the 22 nd Annual international solid freeform fabrication symposium, 348-362

6. Calignano F (2014) Design optimization of supports for overhanging structures in aluminum and titanium alloys by selective laser melting. Mater Des 64:203-213. doi:10.1016/j.matdes.2014.07.043

7. Chahine G, Smith P, Kovacevic R (2010) Application of topology optimization in modern additive manufacturing. Paper presented at the Solid Freeform Fabrication Symposium. Austin, Texas, 606-618

8. Chang K-H, Tang P-S (2001) Integration of design and manufacturing for structural shape optimization. Adv Eng Softw 32(7):555567
9. Kranz J, Herzog D, Emmelmann C (2015) Design guidelines for laser additive manufacturing of lightweight structures in TiAl6V4. J Laser Appl 27(S1):S14001. doi:10.2351/1.4885235

10. Leary M, Babaee M, Brandt M, Subic A (2013) Feasible build orientations for self-supporting fused deposition manufacture: a novel approach to space-filling Tesselated geometries. Adv Mater Res 633:148-168. doi:10.4028/www.scientific.net/AMR.633.148

11. Leary M, Merli L, Torti F, Mazur M, Brandt M (2014) Optimal topology for additive manufacture: a method for enabling additive manufacture of support-free optimal structures. Mater Des 63:678690. doi:10.1016/j.matdes.2014.06.015

12. Lee Y-S, González JA, Lee JH, Kim YI, Park KC, Han S (2016) Structural topology optimization of the transition piece for an offshore wind turbine with jacket foundation. Renew Energy 85: 1214-1225. doi:10.1016/j.renene.2015.07.052

13. Lynch ME, Gu W, El-Wardany T, Hsu A, Viens D, Nardi A, Klecka M (2013) Design and topology/shape structural optimisation for additively manufactured cold sprayed components. Virtual and Physical Prototyping 8(3):213-231. doi:10.1080/17452759.2013.837629

14. Materialise (2016) 3D Printing Software, http://software. materialise.com/magics. Accessed 25 June 2016

15. Seabra L M R (2015) Design optimisation for selective laser melting (SLM) and experimental testing of an aircraft component

16. Strano G, Hao L, Everson RM, Evans KE (2012) A new approach to the design and optimisation of support structures in additive manufacturing. Int J Adv Manuf Technol 66(9-12):1247-1254. doi:10.1007/s00170-012-4403-x

17. Thomas D (2009) The development of design rules for selective laser melting. PhD Thesis. University of Wales

18. Tomlin M, Meyer J (2011) Topology optimization of an additive layer manufactured (ALM) aerospace part. Paper presented at the The 7th Altair CAE Technology Conference, Gaydon, UK, 10th May, 1-9

19. Wang D, Yang Y, Liu R, Xiao D, Sun J (2013) Study on the designing rules and processability of porous structure based on selective laser melting (SLM). J Mater Process Technol 213(10):17341742. doi:10.1016/j.jmatprotec.2013.05.001

20. Xiao D-m, Yang Y-q, Su X-b, Di W, Luo Z-y (2012) Topology optimization of microstructure and selective laser melting fabrication for metallic biomaterial scaffolds. Trans Nonferrous Metals Soc China 22(10):2554-2561

21. Zegard T, Paulino GH (2015) Bridging topology optimization and additive manufacturing. Struct Multidiscip Optim 53(1):175-192. doi:10.1007/s00158-015-1274-4

22. Zhang Y, Bernard A, Gupta RK, Harik R (2014) Evaluating the design for additive manufacturing: a process planning perspective. Procedia CIRP 21:144-150. doi:10.1016/j.procir.2014.03.179 